\title{
ESTADO DA ARTE NO TRATAMENTO DO CÂNCER DE PRÓSTATA METASTÁTICO
}

\section{ARTIGO ORIGINAL}

VELLOSO, Leonardo Pompeu Leão ${ }^{1}$

CUNHA, Gustavo Rodrigues ${ }^{2}$

SILVA, Rafael Vargas ${ }^{3}$

PEREIRA, Nalisson Marques ${ }^{4}$

VELLOSO, Leonardo Pompeu Leão. Et al. Estado da arte no tratamento do câncer de próstata metastático. Revista Científica Multidisciplinar Núcleo do Conhecimento. Ano 05, Ed. 08, Vol. 02, pp. 52-71. Agosto de 2020. ISSN: 2448-0959, Link de acesso: https://www.nucleodoconhecimento.com.br/saude/prostata-metastatico

\section{RESUMO}

O câncer de próstata é a segunda causa mais frequente de morte e o tipo de neoplasia maligna mais diagnosticada em homens adultos no mundo. Seu manejo está evoluindo à medida que a estratificação de risco melhora, tendo-se, em casos selecionados, a possibilidade de monitoramento seguro mediante vigilância ativa, já em outros, condutas mais invasivas através de terapia cirúrgica, radiação ou braquiterapia, buscando-se extirpar as células cancerígenas antes que atinjam a

${ }^{1}$ Acadêmico do $11^{\circ}$ período do curso de medicina pela Universidade Federal do Amapá (UNIFAP).

${ }^{2}$ Acadêmico do 9ํㅜ período do curso de medicina pela Universidade Federal do Amapá (UNIFAP).

${ }^{3}$ Acadêmico do 9ำ período do curso de medicina pela Universidade Federal do Amapá (UNIFAP).

${ }^{4}$ Docente do Curso de Medicina pela Universidade Federal do Amapá. 
corrente sanguínea e originem metástases à distância. Ademais, na falha da conduta em fase inicial ou em recidiva, com aumento dos níveis de antígeno prostático específico ou surgimento de metástases, a privação androgênica é a base da terapia sistêmica. Nesse instante, contudo, o bloqueio hormonal pode não ser uma opção curativa, uma vez em que, muitos dos tumores de próstata não mais confinados no órgão de origem, em algum momento, apresentarão células cujo crescimento é independente de andrógenos. Para a doença avançada e refratária ao referido tratamento, vê-se pior prognóstico, haja vista que, mesmo com o surgimento de novos medicamentos nos últimos anos, carece-se de opções que controlem a progressão neoplásica a longo prazo. Tal cenário torna coerente o interesse por estudos com novas possibilidades nesse estágio da doença. Dado que pesquisas com resultados promissores em humanos levantam perspectivas para pacientes com câncer de próstata resistente à castração; objetiva-se neste trabalho: selecionar, sistematizar e apresentar trabalhos que tenham obtido resultados promitentes para pacientes com câncer de próstata metastático resistente à castração. Por meio de plataformas digitais, foram encontrados 55 artigos e filtrou-se 13 que cumpriram os critérios de inclusão e exclusão. Pode-se oferecer, mediante tais resultados, um acesso direto e conciso às informações para quando a doença se torna refratária à hormonioterapia.

Palavras-chave: câncer, próstata, metastático, hormônio-refratário, tratamento.

\section{INTRODUÇÃO}

O câncer de próstata traz um ônus global substancial, uma vez em que se encontra entre as cinco principais neoplasias quanto à incidência e mortalidade (FERLAY et al., 2015). Outrossim, faz-se o referido carcinoma, o mais comum e a segunda causa de mortes, por tumores malignos, mais frequente em adultos do sexo masculino, sobretudo, nos com mais de 65 anos de idade, os quais correspondem a $64 \%$ dos novos casos de pacientes diagnosticados com a doença (KESSLER et al., 2012) (VON EYBEN et al., 2018). No ano de 2015, a neoplasia maligna da próstata foi diagnosticada em 1.600.000 pessoas, bem como foi responsável por 366.000 mortes (CAMPS et al., 2018). 
No que diz respeito ao desenvolvimento da referida enfermidade, o adenocarcinoma que deriva de tecido glandular é responsável pela maioria das neoplasias, as quais geralmente progridem localmente antes de se tornarem metastáticas (NELSON et al., 2003).

A presença de áreas nodulares ou assimétricas, ao exame de próstata, ou um nível elevado de Antígeno Prostático Específico (PSA), ao exame de sangue, levantam a possibilidade de que o paciente esteja acometido pela doença (MCCLURE et al., 2018). Há, neste caso, para pacientes com expectativa de vida maior que 10 anos ou com sintomas de metástases, indicação para biópsia da próstata a fim de confirmar a suspeita clínica e dar início ao tratamento (MCCLURE et al., 2018).

É improvável, quanto ao tratamento dos pacientes acometidos pela supradita neoplasia, que uma única sequência terapêutica seja a ideal para todos e se faz necessária, por conseguinte, uma estratégia individualizada, para cada um, a fim de se aproximar do objetivo de obter a melhor eficácia no combate à doença (LORENTE et al., 2016). Não obstante, permanece, o câncer de próstata metastático resistente à castração (CPMRC), uma enfermidade fatal (SCHWEIZER et al., 2012).

No momento em que se chega nesta etapa do tratamento, com o câncer de próstata metastático (CPM) resistente à castração, é quando as respostas terapêuticas atuais não mais conseguem apresentar boas taxas de remissão e a taxa de Sobrevida Media (SVM) caí muito com grande dificuldade de sequer ter-se bons biomarcadores para guiar a decisão terapêutica (KARANTANOS et al., 2013). Visto isso, diversos estudos estão sendo desenvolvidos em busca tanto de opções de tratamento, quanto de parâmetros para melhorar a decisão terapêutica, haja vista que ainda não se tem algo definitivamente consolidado em nenhuma das duas vertentes (NUHN et al., 2019). Vislumbrando esse fato, entendendo toda a sistemática da intervenção que é consolidada até esse momento e considerando que ainda não existe uma opção definitiva nessa etapa da terapia, é possível concluir a partir da absência de informação cientificamente estável que esse momento terapêutico é a conjuntura que define o estado da arte no tratamento do CPM. 


\subsection{JUSTIFICATIVA}

A partir do momento em que o cancro de próstata se apresenta refratário às opções terapêuticas já consolidadas, tornam-se as terapias paliativas a única opção de tratamento (SACHPEKIDIS et al., 2019). Outrossim, muitas novidades, para o combate à supradita neoplasia, quando já refratária à castração, vêm sendo apresentadas em diversos lugares do mundo (NUHN et al., 2019), todavia pouca organização do assunto é vista na literatura nacional, já que ao se pesquisar as palavras-chave "câncer", "próstata" e "metástase" na SciELO encontra-se apenas dois resultados de estudos que discorrem sobre a temática (TALBOT et al., 2008) (KALIKS et al., 2010).

\subsection{OBJETIVO}

Neste estudo objetiva-se auxiliar os especialistas e estudiosos da temática a fazerem a sistematização concisa e crítica do saber vigente por meio da apresentação organizada de pesquisas promissoras desenvolvidas sobre o CPM. Para isso, propõese, viabilizar em língua portuguesa, uma revisão do assunto que possibilite o rápido acesso ao conhecimento acerca de terapias capazes de beneficiar pacientes com CPM. Fazendo isso, busca-se ampliar o leque terapêutico para os que estejam em estágio terminal da doença, de forma que esses tenham opções além do tratamento exclusivamente paliativo a partir do momento em que ocorra resposta insatisfatória às intervenções classicamente consolidadas na literatura.

\section{DESENVOLVIMENTO}

Ao se considerar um paciente diagnosticado e devidamente assistido desde o início do câncer de próstata localizado, tem-se, como as opções de tratamento: radioterapia externa, braquiterapia ou prostatectomia; todas com o escopo de eliminação das células cancerígenas (CHEN, 2017). Ademais, a depender da agressividade do referido tumor, pode-se ter como opção, a observação vigilante da doença e seu padrão de evolução (SUREDA, 2019). 
Caso não se atenda ainda o objetivo de redução do PSA a níveis desejáveis ou ocorra recorrência bioquímica da neoplasia prostática maligna, tem-se a opção de iniciar a terapia antiandrogênica mediante orquiectomia bilateral, análogos do hormônio liberador do hormônio luteinizante, estrógenos ou antiandrógenos (DENIS et al., 2000). Essa conduta, por meio da diminuição da produção de testosterona, pode oferecer redução da doença, uma vez em que as células cancerígenas são provenientes de tecido prostático e dependem do hormônio sexual masculino para sua sobrevivência e multiplicação (STUDENT et al., 2020).

Mesmo com a terapia de supressão androgênica, é possível que as células cancerígenas, já mais indiferenciadas, continuem a se desenvolver (SHANKAR et al., 2020). A partir desta apresentação da doença, tem-se, como alternativa, associar o Acetato de Abiraterona, medicamento que impede a produção de testosterona, na medida em que inibe o citocromo P17, o qual se faz fundamental para a gênese do andrógeno em todas as células do organismo (YUAN et al., 2014) (SHANKAR et al., 2020). Outra opção, neste estágio da doença, trata-se da Enzalutamida, droga capaz de inibir a recepção e o efeito do hormônio masculinizante nas células saudáveis ou neoplásicas do paciente (YUAN et al., 2014).

Como outra alternativa ou para quando a doença se torne resistente ao Acetato de Abiraterona e à Enzalutamida, tem-se o tratamento com agentes quimioterápicos, Docetaxel (primeira linha) e Cabazitaxel (segunda linha) para o CPMRC (GEORGE et al., 2020).

\subsection{METODOLOGIA}

Trata-se de uma revisão bibliográfica sistemática integrativa. A busca dos artigos consistiu em consulta às seguintes bases de periódicos: LILACS, PubMed e SciELO.

Foram utilizados os seguintes critérios de inclusão: i) estudos resultados da busca com as palavras chaves "remission metastatic castration resistant prostate" e "câncer próstata metastático resistente castração remissão" nas supracitadas plataformas; ii) estudos com o dado de SVM ou que através dos dados apresentados tivessem o seu 
cálculo possível de ser feito; iii) estudos em que os pacientes do grupo de intervenção tiveram SVM maior ou igual a 10 meses, esse valor foi o ponto de corte devido ao fato de estar próximo da SVM em pacientes que utilizaram o Cabazitaxel, a terapia usual. Acerca das palavras chaves, foi escolhido "câncer", "próstata", "metastático", "resistente" e "castração" com o intuito de selecionar o momento do paciente em que as terapias possíveis são muitas e onde mais se identifica o atual estado da arte no tratamento do câncer de próstata e "remissão" foi escolhido com o intuito de se ter um dado qualitativo sobre a resposta do paciente em relação à intervenção utilizada.

Os critérios de exclusão foram: i) estudos com câncer hormônio sensível; ii) estudos com objetos de intervenção não humanos; iii) estudos que não avaliaram a eficácia do tratamento em um grupo de pacientes; iv) relatos de caso, devido ao fato desses não relatarem a sobrevida em prospecção dos pacientes, deturpando a SVM dos pacientes; v) estudos de retratamento, devido ao fato de que esses não apresentam a SVM do grupo de tratamento inicial, deturpando esse dado. Dos 55 artigos inicialmente encontrados, 13 passaram pelos critérios descritos.

A partir disso, os artigos foram lidos na íntegra e os pacientes conjugados em grupos de acordo com as terapias descritas nos estudos, dentro de cada grupo conjugado, foi refeito o cálculo de SVM através da media proporcional de acordo com o total de participantes, integrando-se os artigos que possuíssem terapias equivalentes. Seguidamente, foi feito o cálculo do produto de validade (PV) para cada terapia, definido pela multiplicação do número de estudos publicados pela posição no ranque crescente da quantidade de pacientes presentes, sendo a terapia com maior quantidade de pacientes ocupando a posição 15 e a com menor quantidade pacientes a posição 1, por fim, multiplicou-se o resultado obtido pela SVM proporcional previamente calculada.

Então, através do Excel, montou-se um gráfico, utilizando-se como parâmetro para ordenação o PV calculado, um gráfico utilizando-se como parâmetro para ordenação a SVM e uma tabela com os dados obtidos utilizando-se como parâmetro para ordenação o PV calculado. Por fim, fez-se a discussão dos gráficos, tabelas e resultados. 


\subsection{RESULTADOS}

Gráfico 1. Valores da Sobrevida Media nos grupos de terapias gerados pela integração dos estudos.

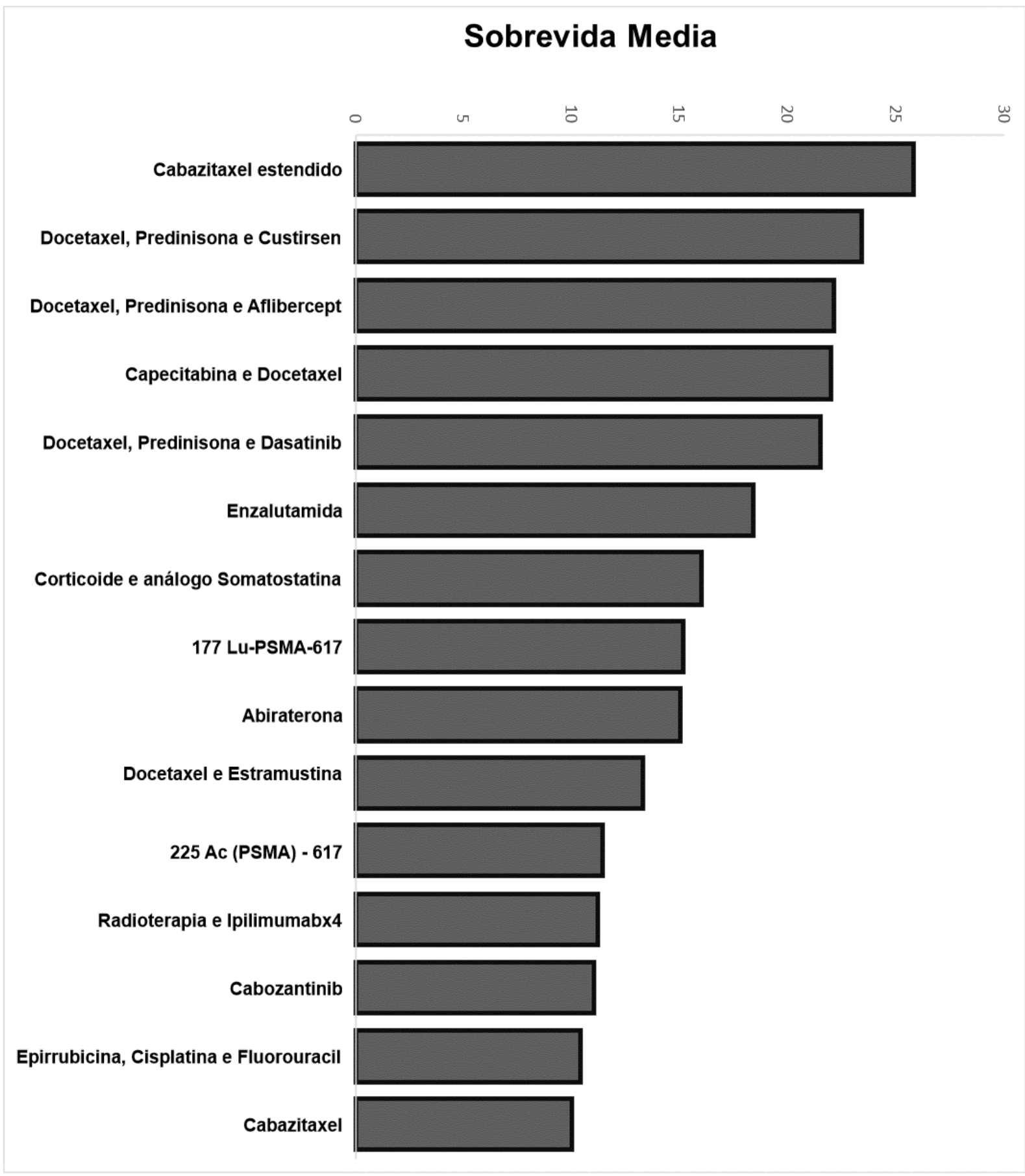

Fonte: Dados da pesquisa. 
Gráfico 2. Valores do Produto de Validade nos grupos de terapias gerados pela integração dos estudos.

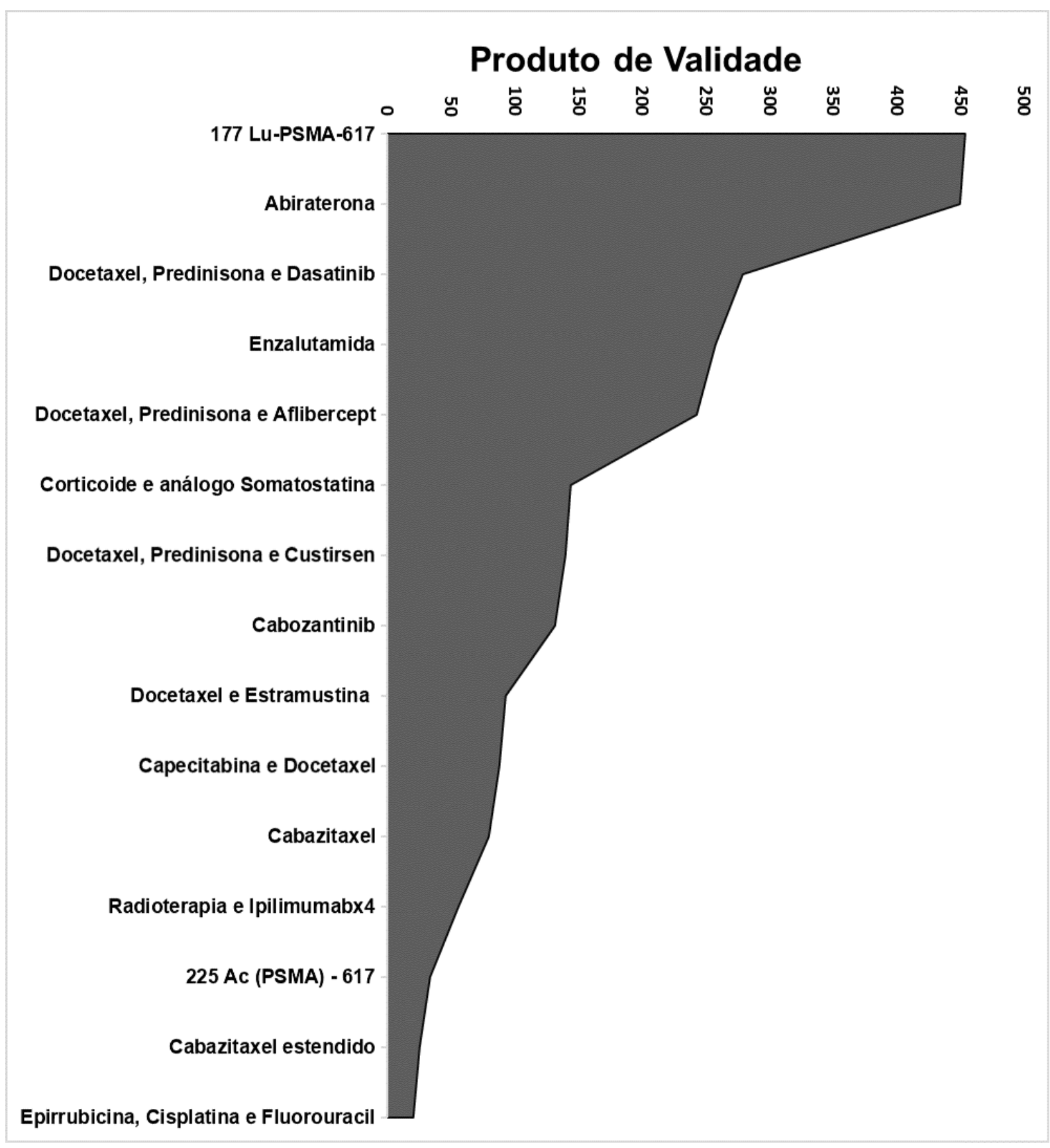

Fonte: Dados da pesquisa.

$\mathrm{Na}$ busca realizada em todas as bases de dados referidas foram encontrados 13 estudos com um total de 5.415 pacientes. A sobrevida média dos pacientes nos trabalhos foi de 16, 4 meses. 
Os dados acerca da SVM foram colocados didaticamente na figura 1 e os dados do PV na figura 2. Pouco pode-se afirmar acerca do intervalo de confiança ou significância estatística a respeito de cada terapia, visto que a maioria deles deu enfoque para outros parâmetros nesse aspecto, como a queda do PSA, por exemplo, que não a sobrevida média. Outros valores prospectados são descritos a seguir conforme a tabela 1.

No tratamento rotulado pelo antígeno prostático específico de membrana 225 ActínioPSMA-617 analisado em um estudo com 17 pacientes $(n=17)$, observou-se remissão das lesões em 15, os quais possuíam avidez das lesões por radiomarcador maior que $50 \%$ no exame de imagem e remissão total em 11 pacientes. Três pacientes evoluíram a óbito, bem como um decidiu por trocar de terapia e faleceu em 4 meses. Foi relatado pelo estudo 14 vivos em 13 meses de acompanhamento. Houve declínio de PSA maior ou igual a $90 \%$ em $82 \%$ dos pacientes, incluindo 7 pacientes dos quais obtiveram PSA sérico indetectável e remissão de até doze meses após a terapia. Quinze pacientes tiveram avidez das lesões por marcador nas imagens maior que $50 \%$, incluindo os onze que obtiveram resolução completa.

A terapia dirigida com 177 Lutécio-PSMA-617 foi analisada em 3 estudos: dois experimentais e uma revisão sistemática. Na revisão foram incluídos 669 pacientes, dos quais 109 realizaram esse tratamento como primeira linha $(n=109)$ com remissão em 31 deles e declínio do PSA maior ou igual a 50\% em 44\% dos pacientes e SVM de 14 meses. Em um dos estudos experimentais, realizado com 56 pacientes $(n=56)$, obteve-se remissão parcial das lesões em 14 de 25 pacientes analisados, 45 apresentaram declínio do PSA com SVM de 25,3 meses e 12 pacientes faleceram ao decorrer do estudo. No outro estudo experimental houve remissão parcial em $23 \%$ dos pacientes, declínio do PSA maior que $50 \%$ em $45,5 \%$ dos 90 pacientes $(n=90)$ e SVM de 14 meses. A média das SVM foi de 15,1 em um total de 558 pacientes.

Dexametasona e análogo de somatostatina foi vista em uma metanálise de estudos não controlados e apresentou declínio de PSA igual ou maior que $50 \%$ em $59,5 \%$ dos pacientes. Obteve SVM de 16 meses nos nove estudos citados pelo trabalho, somando um total de 87 pacientes $(n=87)$. 
$\mathrm{Na}$ terapia com Docetaxel e fosfato de Estramustina, encontrado em um artigo experimental com 54 pacientes analisados $(n=54)$, um em 24 pacientes teve resposta completa e oito obtiveram resposta parcial, bem como 22 de 49 pacientes apresentaram declínio de PSA. A SVM foi de 13,3 meses.

$\mathrm{Na}$ análise da terapia com Capecitabina e Docetaxel com 30 pacientes $(n=30)$, o declínio do PSA foi igual ou maior que $50 \%$ em $76 \%$ dos pacientes e o declínio igual ou maior que $90 \%$ em $31 \%$ dos pacientes e a SVM obtida foi de 22 meses.

Para o Cabazitaxel estendido, em 3 dos 4 pacientes o PSA teve queda sustentada em até 23 ciclos de uso. Dos quatro pacientes $(n=4)$, três tiveram sobrevida de 29, 30 e 12 meses e um ainda permanecia vivo, assumindo-se para esse, sobrevida mínima de 23,6 meses para o cálculo da SVM do estudo, sendo essa igual a 25,8 meses.

Docetaxel com Prednisona e Aflibercept apresentou SVM de 22,1 meses com 612 ( $\mathrm{n}$ = 612) pacientes em uma revisão sistemática encontrada.

Docetaxel, Prednisona e Dasatinib apresentou SVM de 21,5 meses em dois estudos encontrados em um total de 762 paciente $(n=762)$.

A Enzalutamida apresentou SVM de 18,4 meses em um estudo encontrado em um total de 1199 paciente $(n=1199)$.

Docetaxel associada a Prednisona e Custirsen apresentou a segunda melhor resposta com 23,4 meses de SVM, cujo número de pacientes observados foi de $41(n=41)$ em um estudo analisado.

Cabozantinib apresentou SVM de 11 meses em 1 estudo encontrado em um total de 682 pacientes $(n=682)$.

Associação de Capecitabina e Docetaxel apresentou SVM de 22 meses em um estudo filtrado, cujo número de pacientes analisados foi de $30(n=30)$.

Cabazitaxel apresentou SVM de 10 meses em 1 estudo encontrado com um total de 69 pacientes $(n=69)$. 
Radioterapia e Ipilimumabx4 apresentou SVM de 11,2 meses em 1 estudo encontrado com um total de 34 pacientes $(n=34)$.

Tabela 1. Dados obtidos e tabelados dos grupos de terapias gerados pela integração dos estudos.

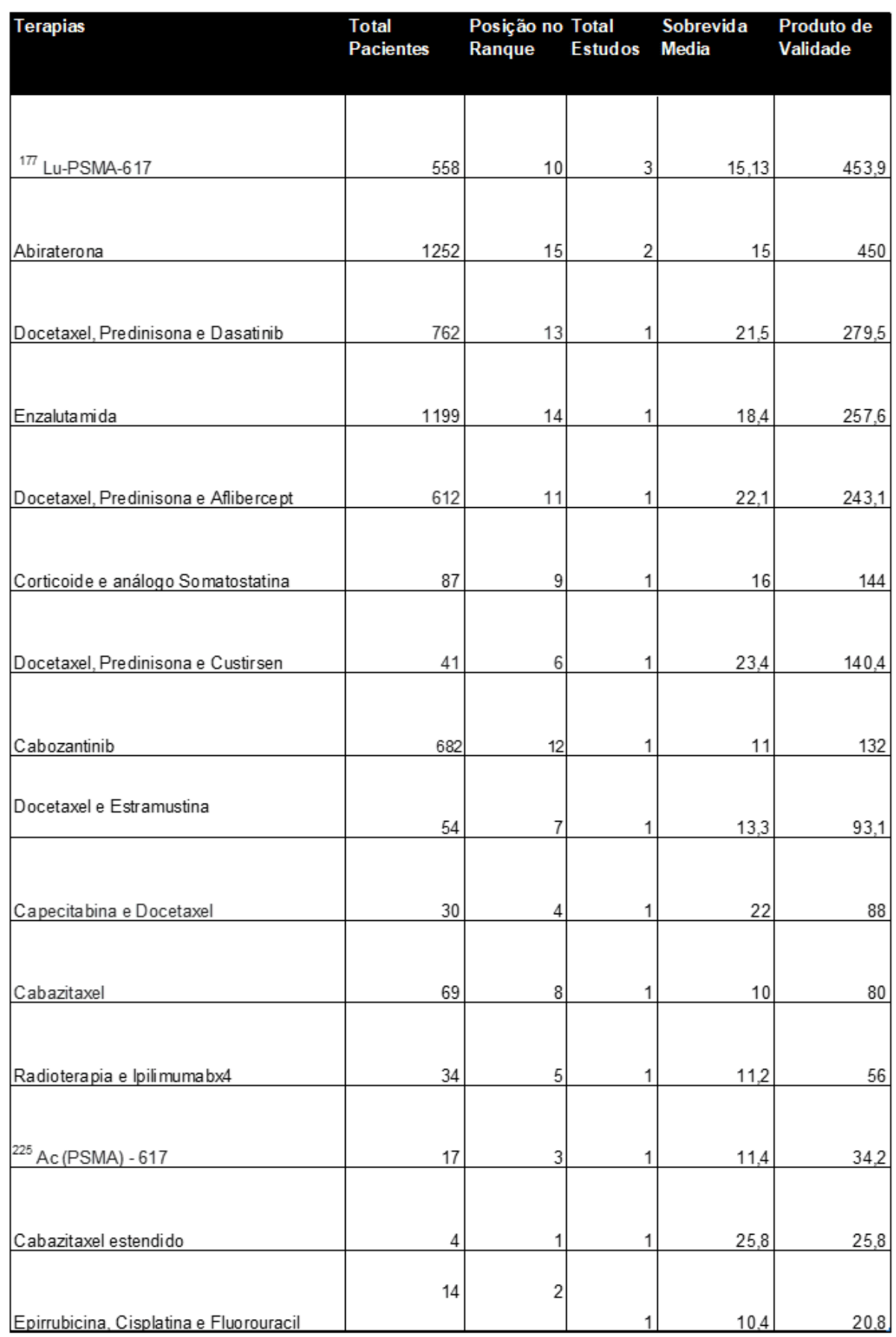

Fonte: Dados da pesquisa.

RC: 57092

Disponível em: https://www.nucleodoconhecimento.com.br/saude/prostata-metastatico 


\subsection{DISCUSSÃO}

Das quinze intervenções analisadas, treze foram encontrados em apenas um estudo, demonstrando a pouca repetição multicêntrica desses e dando o sinal de que têm-se pouca consolidação definitiva de uma melhor terapia dentre as propostas na literatura (MONTEIRO et al., 2019).

O estudo que se destacou em relação à SVM - principal ponto de inflexão deste trabalho - foi o Cabazitaxel estendido, com valor de 25,8 meses, ocupando a primeira posição no ranque de SVM. Contudo, com um total de 4 pacientes, o seu PV foi de 25,8, colocando a terapia em penúltima colocada no ranque desse parâmetro, demonstrando que apesar do bom resultado, a terapia ainda deve ser testada em grupos maiores de pacientes antes de ter-se alguma conclusão mais definitiva, uma vez em que são limitados os dados no que concerne ao uso de mais de 10 ciclos de Cabazitaxel até que ocorra progressão da doença ou intolerância ao quimioterápico (NORONHA et al., 2015).

Outro destaque é a Enzalutamida, pois foi o estudo com o segundo maior espaço amostral dos quinze tratamentos, apresentando-se com 1199 pacientes e SVM de 18,4 meses. Assim, apesar de possuir uma SVM menor que o Cabazitaxel estendido, a Enzalutamida fornece maior grau de consolidação na literatura e destaca-se como uma interessante opção terapêutica no presente momento, corolário compatível com resultados prévios da literatura acerca da eficácia no Câncer de Próstata Resistente à Castração (HUSSAIN et al., 2018), supondo-se, assim, pela somatória dos resultados encontrados e com as conclusões do citado estudo, uma possível extrapolação da eficácia do tratamento também para o CPMRC.

A terapia com Docetaxel, prednisona e dasatinib obteve, em relação ao número de pacientes, a terceira maior soma dentre todos os estudos e a segunda maior dentre os de estudo único. Apesar de ter sido encontrada em apenas um artigo, destacouse, pois obteve uma boa relação entre o número de pacientes e a SVM de 21,5 meses, obtendo a terceira melhor colocação no ranque do PV. Esta sobrevida media deveuse principalmente à combinação de docetaxel e prednisona, pois a adição de dasatinib 
ou outros agentes não demonstrou aumento comprovado do benefício (VAISHAMPAYAN, 2016).

As terapias restantes tiveram desvantagem em relação às citadas anteriormente, cujas SVM, embora fossem maiores, ou possuíam um menor espaço amostral ou perdiam no ranque do PV em comparação com as outras. Portanto, Cabazitaxel estendido, Enzalutamida e Docetaxel com prendnisona associados ao dasatinib foram as opções com os melhores dados quantitativos.

As próxima duas terapias a serem citadas tiveram mais do que um estudo publicado e apresentaram desempenhos semelhantes nos ranques do PV e da SVM. A principal diferença entre ambas foi o total de pacientes.

A terapia dirigida com 177 Lutécio-PSMA-617 obteve o melhor PV entre todos os estudos, impulsionada, sobretudo, por ter 3 estudos prospectados, sendo a intervenção com o maior número de artigos. Foi obtida SVM de 15,3 meses. Resultados positivos compatíveis com a conclusão de estudos de revisão que se posicionaram em prol da citada intervenção a despeito de outros tratamentos de terceira linha (VON EYBEN et al., 2017) (BRÄUER et al., 2017).

Ao se comparar a terapia 225 Actínio-PSMA-617 com 177 Lutécio-PSMA-617, observa-se melhor eficácia no que concerne à redução da doença já nos primeiros ciclos de administração do radiofármaco e, consequentemente, toxicidade reduzida quanto às glândulas salivares. Faz-se notável, ainda, a eficácia terapêutica desse medicamento, haja vista que, mediante seu uso, vê-se possibilidade de alcançar remissão da doença em pacientes com câncer de próstata avançado sem quimioterapia prévia e refratários à hormonioterapia (SATHEKGE et al., 2018), resultado esse, único dentre as quinze opções terapêuticas prospectadas.

A Abiraterona teve destaque pelo maior número de pacientes analisados $(n=1252)$ em dois estudos, demonstrando possível maior significância estatística, o que é compatível com o tempo de vigência da utilização da terapia; encontra-se estudos com pacientes apresentando resultados positivos há mais de dez anos (FIZAZI et al., 
2012). Embora a SVM de 15 meses não seja a mais animadora entre as aqui apresentadas, acredita-se que esse seja um número mais estável se comparado com as outras quatorze intervenções estudadas, perspectiva consoante à de Monteiro (2019), o qual classifica a terapia como de primeira ou de segunda linha no tratamento.

Assim, unificando os dados e argumentos apresentados, é possível concluir que não há consolidação definitiva na literatura acerca de uma principal opção terapêutica e mais estudos são necessários para a validação estatística dos dados. Apesar disso, foram expostas algumas das mais promissoras intervenções vigentes mediante dados qualitativos, comparação e ranqueamento de dados quantitativos que podem ser de grande valia para o médico e o paciente na decisão analítica de qual tratamento é o mais adequado ao caso alvo de acordo com a sua disponibilidade de inserção em protocolo de pesquisa e contexto clínico ou patológico, ideia compartilhada por Monteiro (2019) em sua revisão crítica acerca do assunto.

\section{CONSIDERAÇÕES FINAIS}

Conclui-se, por fim, a partir dos resultados deste estudo, que, em um cenário no qual - CPM se encontra refratário à todas as opções terapêuticas já validadas, tem-se novas perspectivas, em potencial, no que diz respeito a tratamentos que ainda estão em fase de testes. Apesar da incerteza quanto aos ganhos em sobrevida e qualidade de vida mediante tratamentos ainda não consolidados, fazer parte de programas de ensaios clínicos de estudos que já obtiveram bons resultados, mesmo que em um número limitado de pacientes, pode se tornar uma opção tendo em vista alguma possibilidade de ainda se obter remissão, estabilização ou atenuação da velocidade de progressão da neoplasia em questão, bem como maior ganho de sobrevida. Outrossim, mediante esta revisão de literatura, pode-se obter, de maneira rápida, concisa e organizada, informações relevantes quanto aos ganhos de SVM relatados em estudos, possivelmente, promissores no referido cenário de prognóstico sombrio para o cancro de próstata avançado e refratário à privação androgênica. 


\section{REFERÊNCIAS}

BAUM, R. P. et al. 177Lu-labeled prostate-specific membrane antigen radioligand therapy of metastatic castration-resistant prostate cancer: Safety and efficacy. Journal of Nuclear Medicine, Bad Berka, v. 57, n. 7, p. 1006-1013, 1 jul. 2016.

BRÄUER, A. et al. 177Lu-PSMA-617 radioligand therapy and outcome in patients with metastasized castration-resistant prostate cancer. European Journal of Nuclear Medicine and Molecular Imaging, Berlin, v. 44, n. 10, p. 1663-1670, 1 set. 2017.

CAMPS, S. M. et al. The Use of Ultrasound Imaging in the External Beam Radiotherapy Workflow of Prostate Cancer Patients. BioMed Research International, New York, v. 2018, 2018. DOI: 10.1155/2018/7569590. Disponível em: https://www.ncbi.nlm.nih.gov/pmc/articles/PMC5829356/. Acesso em: 30 jul. 2020.

CHEN, R. C. et al. Association Between Choice of Radical Prostatectomy, External Beam Radiotherapy, Brachytherapy, or Active Surveillance and Patient-Reported Quality of Life Among Men With Localized Prostate Cancer. JAMA, Chicago, v. 317, n. 11, p. 1141-1150, 21 mar. 2017.

DENIS, L. J.; GRIFFITHS, K. Endocrine treatment in prostate cancer. In: Seminars in surgical oncology. New York: John Wiley \& Sons, Inc., 2000. p. 52-74.

DI LORENZO, G. et al. Phase II study of docetaxel re-treatment in docetaxelpretreated castration-resistant prostate cancer. BJU International Oxford, v. 107, n. 2, p. 234-239, jan. 2011.

EFSTATHIOU, E. et al. Combination of docetaxel, estramustine phosphate, and zoledronic acid in androgen-independent metastatic prostate cancer: Efficacy, safety, and clinical benefit assessment. Urology, Ridgewood, v. 65, n. 1, p. 126-130, 20 fev. 2012.

EFSTATHIOU, E. et al. Effects of abiraterone acetate on androgen signaling in castrate-resistant prostate cancer in bone. Journal of Clinical Oncology, New York 
v. 30, n. 6, p. 637-643, 20 Fev 2012. DOI: 10.1200/JCO.2010.33.7675. Disponível em: https://pubmed.ncbi.nlm.nih.gov/22184395/. Acesso em: 29 jul 2020.

EYBEN, F. E. et al. Third-line treatment and 177Lu-PSMA radioligand therapy of metastatic castration-resistant prostate cancer: a systematic review. European Journal of Nuclear Medicine and Molecular Imaging, Berlin, v. 45, n. 3, p. 496-508, 1 mar. 2018. DOI: 10.1007/s00259-017-3895-x. Disponível em: https://ink.springer.com/article/10.1007\%2Fs00259-017-3895-x. Acesso em: 30 jul. 2020.

FIZAZI, K. et al. Abiraterone acetate for treatment of metastatic castration-resistant prostate cancer: Final overall survival analysis of the COU-AA-301 randomised, double-blind, placebo-controlled phase 3 study. The Lancet Oncology, London, v. 13, n. 10, p. 983-992, 2012. DOI: https://doi.org/10.1016/S1470-2045(12)70379-0. Disponível em: https://www.thelancet.com/jAAournals/lanonc/article/PIIS14702045(12)70379-0/fulltext\#secd29650609e2060. Acesso em: 30 jul. 2020.

GEORGE, D. J. et al. Treatment Patterns and Outcomes in Patients With Metastatic Castration-resistant Prostate Cancer in a Real-world Clinical Practice Setting in the United States. Clinical Genitourinary Cancer, Dallas, v. 0, n. 0, p. 2020-2021, 7 jan. 2020.

GUPTA, S. et al. Ecf chemotherapy for liver metastases due to castration-resistant prostate cancer. Journal of the Canadian Urological Association, Montréal, v. 8, n. 9, p. 353-357, 1 out. 2014.

HUSSAIN, M. et al. Enzalutamide in Men with Nonmetastatic, Castration-Resistant Prostate Cancer. New England Journal of Medicine, Boston, v. 378, n. 26, p. 24652474, 2018. DOI: 10.1056/NEJMoa1800536. Disponível em: https://www.nejm.org/doi/full/10.1056/NEJMoa1800536. Acesso em: 30 jul. 2020.

IHDE, D. C. et al. Effective treatment of hormonally-unresponsive metastatic carcinoma of the prostate with adriamycin and cyclophosphamide methods of 
documenting tumor response and progression. Cancer, Washigngton, v 45, n. 6, p. 1300-1310, 15 mar.1980.

KALIKS, R. A. et al. Complete androgen blockade safely allows for delay of cytotoxic chemotherapy in castration refractory prostate cancer. International Braz J Urol, Rio de Janeiro, v. 36, n. 3, p. 1677-5528, jun. 2010.

KARANTANOS, T.; CORN, P. G.; THOMPSON, T. C. Prostate cancer progression after androgen deprivation therapy: Mechanisms of castrate resistance and novel therapeutic approaches. Oncogene, Houston, v. 32, n. 49, p. 5501-5511, 10 jun. 2013.

KESSLER, E. R.; FLAIG, T. W. Optimal management of recurrent prostate cancer in older patients. Drugs and Aging, v. 29, n. 11, p. 871-883, 8 nov. 2012.

LORENTE, D. et al. Optimal Treatment Sequence for Metastatic Castration-resistant Prostate Cancer. European Urology Focus, Amsterdam, v. 2, n. 5, p. 488-498, 1 dez. 2016.

MCCLURE, T. et al. Prostate cancer. In: Encyclopedia of Endocrine Diseases. [s.l.] Elsevier, 2018. p. 784-792.

MONTEIRO, F. et al. Treatment of Advanced Prostate Cancer: Where Are We in 2019?. Brazilian Journal of Oncology, São Paulo, v. 15, n. 0, p. 1-19, 13 mar. 2019.

NELSON, William G.; DE MARZO, Angelo M.; ISAACS, William B. Prostate cancer. New England Journal of Medicine, Boston, v. 349, n. 4, p. 366-381, 24 jul. 2003.

NORONHA, V.; JOSHI, A.; PRABHASH, K. Beyond ten cycles of cabazitaxel for castrate-resistant prostate cancer. Indian Journal of Cancer, Bombay, v. 51, n. 3, p. 363-365, 1 Jul 2014. DOI: 10.4103/0019-509X.146721. Disponível em: $<$ https://pubmed.ncbi.nlm.nih.gov/25494139/>. Acesso em: 28 jul 2020.

NUHN, P. et al. Update on Systemic Prostate Cancer Therapies: Management of Metastatic Castration-resistant Prostate Cancer in the Era of Precision Oncology. 
European Urology, Heidelberg, v. 75, n. 1, p. 88-99, 1 jan. 2019. doi: 10.1016/j.eururo.2018.03.028.

<https://doi.org/10.1016/j.eururo.2018.03.028>. Acesso em: 28 jul 2020.

OUDARD, S. et al. Docetaxel rechallenge after an initial good response in patients with metastatic castration-resistant prostate cancer. BJU International, Oxford, v. 115, n. 5, p. 744-752, 1 Mai 2015. doi: 10.1111/bju.12845. Disponível em: <https://pubmed.ncbi.nlm.nih.gov/24947139/>. Acesso em: 29 jul 2020.

RAGHAVAN, D. First-line use of novel hormonal agents in prostate cancer: A critical appraisal. Clinical Advances in Hematology and Oncology, New York, v. 16, n. 4, p. 289-295, abr. 2018.

SACHPEKIDIS, C. et al. PSMA radioligand therapy in prostate cancer: Overview, latest advances and remaining challenges. Immunotherapy. London, v. 11, n. 15, p. 12671271, 9 set. 2019.

SATHEKGE, M. et al. 225Ac-PSMA-617 in chemotherapy-naive patients with advanced prostate cancer: a pilot study. European Journal of Nuclear Medicine and Molecular Imaging. Amsterdam, v. 46, n. 1, p. 129-138, set. 2019.

SCHWEIZER, M.; ANTONARAKIS, E. Abiraterone and other novel androgen-directed strategies for the treatment of prostate cancer: A new era of hormonal therapies is born. Therapeutic Advances in Urology. Los Angeles, v. 4, n. 4, p. 167-178, ago. 2012.

SHAH, B.; SHRESTHA, S. Durable complete remission with abiraterone in castration resistant metastatic prostate cancer. Acta Oncologica. Stockholm, v. 52, n. 1, p. 174, 05 nov. 2012.

SHANKAR et al. Novel approach to therapeutic targeting of castration-resistant prostate cancer. Medical Hypotheses. Amsterdam, v. 140, n. 109639, 19 fev. 2020. 
SIDDIQUI, K. et al. Role of estrogens in the secondary hormonal manipulation of hormone refractory prostate cancer. Journal of the Pakistan Medical Association. Karachi, v. 54, n. 9, p. 445-447, set. 2004.

STUDENT, S. et al. Anti-androgen hormonal therapy for cancer and other diseases. European Journal of Pharmacology. Amsterdam, v. 866, n. 172783, 5 jan. 2020.

SUREDA. A. et al. Health-related quality of life in men with prostate cancer undergoing active surveillance versus radical prostatectomy, external-beam radiotherapy, prostate brachytherapy and reference population: a cross-sectional study. Health Qual Life Outcomes, London, v. 17, n. 1, p. 1-9, 14 jan. 2019.

TALBOT, J.; CHEVALME, Y. Current evaluation of the clinical utility of fluoromethylcholine-(18F) PET/CT in prostate cancer. Brazilian Archives of Biology and Technology. Curitiba, v. 51, n. spe, p. 71-75, dez. 2008.

TOULIS, K. et al. Dexamethasone plus somatostatin-analog manipulation as bone metastasis microenvironment-targeting therapy for the treatment of castrationresistant prostate cancer: A meta-analysis of uncontrolled studies. Anticancer Research. Highlands, v. 32, n. 8, 3283-3289, ago. 2012.

UDOVICICH, C. et al. 68Ga-prostate-specific membrane antigen-positron emission tomography/computed tomography in advanced prostate cancer: Current state and future trends. Prostate International. Seoul, v. 5, n. 4, p. 125-129, 24 fev. 2017.

VAISHAMPAYAN, U. et al. Phase II Trial of Capecitabine and Weekly Docetaxel for Metastatic Castrate Resistant Prostate Cancer. Journal of Urology. Linthicum, v. 182, n. 1, p. 317-323, jul. 2009.

VAISHAMPAYAN, U. Sequences and combinations of multifaceted therapy in advanced prostate cancer. Current Opinion in Oncology. London, v. 27, n. 3, p. 201208, 27 mai. 2015. 
YADAV, M. et al. Efficacy and Safety of 177Lu-PSMA-617 Radioligand Therapy in Metastatic Castration-Resistant Prostate Cancer Patients. Clinical nuclear medicine. Reston, v. 45, n. 1, p. 19-31 jan. 2020.

YUAN, X. et al. Androgen receptor functions in castration-resistant prostate cancer and mechanisms of resistance to new agents targeting the androgen axis. Oncogene. Stuttgart, v. 33, n. 22, p. 2815-2825, 29 mai. 2014.

ZSCHÄBITZ, S. et al. Efficacy of cabazitaxel treatment in metastatic castration resistant prostate cancer in second and later lines. An experience from two German centers. Journal of Cancer. Sydney, v. 8, n. 4, p. 507-512, 11 fev. 2017.

Enviado: Julho, 2020.

Aprovado: Agosto, 2020. 